of more damage over time) than patients treated conventionally.

Infliximab and similar agents might slow the progression of chronic radiographic changes in AS, although disease progression does not seem to be completely inhibited. Larger studies are required to support these findings.

Katherine Sole

Original article Baraliakos X et al. (2005) Radiographic progression in patients with ankylosing spondylitis after 2 years of treatment with the tumour necrosis factor $\alpha$ antibody infliximab. Ann Rheum Dis 64: 1462-1466

\section{Risedronate, alendronate, and strontium ranelate reduce nonvertebral osteoporotic fracture risk}

To date, evidence that treatments for osteoporosis reduce the risk of nonvertebral fractures has mainly come from post-hoc subgroup analyses. International guidelines state, however, that subgroup analyses should not be the sole basis for conclusions on treatment efficacy. A new systematic review and meta-analysis by Boonen et al. aimed to overcome the limitations of earlier such analyses by stringent selection of trials that evaluated nonvertebral fracture risk in the intention-to-treat analysis, and that met the regulatory registration criteria for osteoporosis treatments.

The authors identified 11 phase III, randomized, placebo-controlled efficacy trials of $\geq 3$ years' duration. Statistically significant reductions in the risk of nonvertebral fracture were seen in three trials: two with risedronate $(P \leq 0.03)$ and one with strontium ranelate $(P=0.04)$. Data from the six eligible bisphosphonate trials were then pooled; a meta-analysis showed that both alendronate and risedronate significantly reduced the relative risk of nonvertebral fracture (alendronate, $P=0.012$; risedronate, $P=0.001$ ).

The results of meta-analyses should not be used to compare the efficacies of different treatments, say the authors, because disease severity, patient demographics and definitions of nonvertebral fracture differ between trials. Not all trials included fractures caused by trauma, for example. There is a need for trials that compare different osteoporosis treatments directly, and that have prevention of nonvertebral fracture as a primary endpoint.

\section{Caroline Barranco}

Original article Boonen S et al. (2005) Effect of osteoporosis treatments on risk of non-vertebral fractures: review and meta-analysis of intention-to-treat studies. Osteoporos Int 16: 1291-1298

\section{Primary Sjögren's syndrome and pregnancy outcome}

Primary Sjögren's syndrome (pSS) is a systemic autoimmune syndrome that results primarily in inflammation and destruction of the salivary and lacrimal glands, but also affects other organs such as the liver, kidney and pancreas. The impact of maternal pSS on pregnancy outcome is unclear, as studies addressing pregnancy outcome have produced conflicting results.

This single-center, case-controlled study aimed to clarify this issue. Of 63 consecutive women with pSS who were approached, 58 agreed to participate in the study; however, two of these had never been pregnant. Questionnaires including demographic and pregnancy-related questions were completed by all 58 patients and by 157 matched controls randomly selected from the Medical Birth Registry of Norway. Additional information about pregnancy outcome was obtained from the Medical Birth Registry for 36 patients, and for the 93 controls matched to those patients.

The average age of onset of pSS was 51.3 years; only four patients were diagnosed before their last pregnancy. No significant difference in pregnancy outcome was found between patients and controls, although two patients with ANTI-SSA AND ANTI-SSB ANTIBODIES had a child with congenital heart block. It is unknown, however, how many patients were demonstrated to have these antibodies, or whether the antibodies were present during pregnancy. The authors conclude that, before disease onset, pSS has no impact on pregnancy outcome, although they noted the potential association of anti-SSA and anti-SSB antibodies with congenital heart block.

Rebecca Doherty

Original article Haga H-J et al. (2005) Pregnancy outcome in patients with primary Sjögren's syndrome. A case-control study. J Rheumatol 32: 1734-1736

\section{GLOSSARY}

ANTI-SSA AND ANTI-SSB ANTIBODIES

Antibodies against ribonucleoproteinic complexes Ro/SSA and $\mathrm{La} / \mathrm{SSB}$ that are commonly detected in patients with Sjögren's syndrome; of these, anti-SSB antibodies are more disease-specific 